\title{
Population pharmacokinetics analysis of nivolumab in Asian and non- Asian patients with gastric and gastro-esophageal junction cancers
}

\author{
Mayu Osawa ${ }^{1} \cdot$ Mayumi Hasegawa ${ }^{1} \cdot$ Akintunde Bello $^{2} \cdot$ Amit Roy $^{2} \cdot$ Matthew W. Hruska $^{2}$
}

Received: 10 December 2018 / Accepted: 5 January 2019 / Published online: 21 January 2019

(c) The Author(s) 2019

\begin{abstract}
Purpose Nivolumab monotherapy provided clinically meaningful antitumor activity in Asian and non-Asian patients with chemotherapy-refractory gastric cancer (GC) or gastro-esophageal junction cancer (GEJC) in the ATTRACTION-2 and CheckMate 032 studies, respectively. This analysis assessed the population pharmacokinetics (PopPK) of nivolumab, the impact of covariates on pharmacokinetics (PK), and the PK of nivolumab flat dosing in GC/GEJC using samples from these studies.

Methods PopPK analyses were conducted using data from 1302 patients with solid tumors, including 387 patients with GC/GEJC who had received nivolumab $3 \mathrm{mg} / \mathrm{kg}$ once every 2 weeks (Q2W). The impact of covariates on nivolumab PK was assessed in the full model. Nivolumab exposures following a flat dose of $240 \mathrm{mg}$ Q2W in patients with GC/GEJC were simulated and compared with those of $3 \mathrm{mg} / \mathrm{kg}$ Q2W.

Results Nivolumab PK was described using a 2-compartment, zero-order intravenous infusion and time-varying clearance (CL) model. Baseline CL in patients with GC/GEJC was $\sim 33 \%$ greater than in patients with non-small cell lung cancer (NSCLC) in second line or subsequent lines of treatment $(2 \mathrm{~L}+)$. The effect of race was not clinically relevant $(<20 \%$ difference). Nivolumab exposures following $240 \mathrm{mg}$ Q2W were similar to $3 \mathrm{mg} / \mathrm{kg}$ Q2W in non-Asian patients and 46\% higher in Asian patients due to lower body weight.

Conclusions Nivolumab CL was increased in GC/GEJC relative to NSCLC 2L+. Higher nivolumab exposures achieved with $240 \mathrm{mg}$ Q2W in Asian patients are predicted to be below the acceptable safety margin, supporting the use of a flat dose in both patient populations.
\end{abstract}

Keywords Nivolumab · Population pharmacokinetics · Gastric cancer · Gastro-esophageal junction cancer

\section{Introduction}

Gastric cancer (GC) is the fifth most common malignancy and the third leading cause of cancer-related death worldwide, with the highest incidence and mortality observed in Asian countries [1]. Current standard-of-care treatments for patients with unresectable, locally advanced, recurrent or metastatic GC, and gastro-esophageal junction cancer

Electronic supplementary material The online version of this article (https://doi.org/10.1007/s00280-019-03771-z) contains supplementary material, which is available to authorized users.

Mayu Osawa

Mayu.Osawa@bms.com

Bristol-Myers Squibb K.K., Tokyo, Japan

2 Bristol-Myers Squibb, Princeton, NJ, USA
(GEJC) who progressed after prior chemotherapy include the anti-vascular endothelial growth factor receptor 2 antibody ramucirumab in combination with paclitaxel, or single agent paclitaxel, docetaxel, or irinotecan [2, 3]. Although these therapies provide a modest survival benefit [4-6], the majority of patients experience disease progression. The third-line treatment options are limited to select types of gastro-esophageal cancers [2, 3], emphasizing the need for new therapies for patients with advanced GC/GEJC.

Nivolumab is a fully human immunoglobulin G4 monoclonal antibody that binds to the programmed death-1 (PD1) membrane receptor [7]. Nivolumab has demonstrated efficacy across a number of solid tumor types and has been approved for various indications [8] in multiple countries worldwide, including in patients with unresectable advanced or recurrent GC that has progressed after chemotherapy [9-12]. 
The efficacy and safety of nivolumab in patients with GC or GEJC have been investigated in two studies, ATT RACTION-2 (ClinicalTrials.gov: NCT02267343) and CheckMate032 (ClinicalTrials.gov: NCT01928394). ATT RACTION-2 was a randomized phase 3 trial that evaluated nivolumab $(n=330)$ vs placebo $(n=163)$ in Asian patients with unresectable advanced or recurrent GC or GEJC who had received $\geq 2$ prior chemotherapy regimens [13]. In the ATTRACTION-2 study, nivolumab $3 \mathrm{mg} / \mathrm{kg}$ administered once every 2 weeks (Q2W) demonstrated superior overall survival (OS) compared with placebo [5.3 months and 4.1 months, respectively, hazard ratio (HR) 0.63 ; $95 \%$ confidence interval (CI) $0.5-0.8 ; P<0.0001]$, and a manageable safety profile [13]. Based on these data, nivolumab was approved in Japan, Korea, Taiwan, and Switzerland for patients with unresectable advanced or recurrent GC that has progressed after chemotherapy [9-12]. In the phase 1/2 CheckMate 032 trial, nivolumab monotherapy $(3 \mathrm{mg} /$ kg Q2W) demonstrated clinically meaningful antitumor activity, durable responses, encouraging long-term OS, and manageable safety in patients $(n=59)$ from the United States and Europe who had chemotherapy-refractory, locally advanced or metastatic GC, GEJC, or esophageal adenocarcinoma [14]. With median follow-up duration of 28 months (range 17-35 months), the investigator-assessed objective response rate was $12 \%$, median duration of response was 7.1 months (95\% CI 3.0-13.2), 12-month survival rate was $39 \%$, and median OS was 6.2 months (95\% CI 3.4-12.4). No clinically meaningful differences were noted in the safety profiles of nivolumab in the ATT RACTION-2 and CheckMate 032 studies [15], and the antitumor activity of nivolumab was consistent between the CheckMate 032 and ATTRACTION-2 studies [13, 14].

The pharmacokinetics (PK) of nivolumab have been characterized previously in non-small cell lung cancer (NSCLC), renal cell carcinoma (RCC), and melanoma, using population $\mathrm{PK}$ (PopPK) analysis in which nivolumab PK were described by a linear two-compartment model with time-varying clearance (CL) [16]. Covariates such as baseline body weight (BW), baseline performance status (PS), baseline estimated glomerular filtration rate (eGFR), age, sex, race, tumor type, tumor burden, and hepatic function had a statistically significant effect on nivolumab CL, but the effects of all these covariates, with the exception of BWT, were within $\pm 20 \%$ of the reference value [16]. In addition, nivolumab has been administered up to $10 \mathrm{mg} / \mathrm{kg}$ Q2W, with a relatively flat exposure response for both safety and efficacy. In particular, OS and the risk of adverse events leading to discontinuation or death were not associated with nivolumab exposure for doses ranging from 1 to $10 \mathrm{mg} / \mathrm{kg}$ Q2W in patients with NSCLC [17] or 0.1 to $10 \mathrm{mg} / \mathrm{kg}$ Q2W in patients with melanoma [18].
Previous investigations of other monoclonal antibodies used in the treatment of GC have determined that the CL of those drugs was greater than the CL of the same drug in other tumor types. For example, when bevacizumab or trastuzumab was administered to patients with GC, the CL estimates were faster than estimates for other solid tumor types $[19,20]$. Therefore, it was important to understand the PK of nivolumab following administration of a nivolumab $3 \mathrm{mg} / \mathrm{kg}$ Q2W-dosing regimen in patients with GC/GEJC.

The purpose of the current analyses was to understand the PK of nivolumab in patients with GC/GEJC, assess the PK in Asian and non-Asian patients, and, through model application, understand the predicted exposures from flat dose nivolumab to support the use of the $240 \mathrm{mg}$ dose Q2W for treating patients with GC/GEJC.

\section{Materials and methods}

\section{Included studies, assays, and data analysis}

The PopPK analysis was performed using data from patients with solid tumors who were treated with nivolumab monotherapy $0.1-10 \mathrm{mg} / \mathrm{kg}$ (single dose or Q2W) in 9 studies (Supplemental Table 1), and for whom, nivolumab serum concentration data were available. Nivolumab monotherapy data were available from 3 phase 1 studies (CA209001, CA209003, and ONO-4538-01), 1 phase 1/2 study (CheckMate 032), 2 phase 2 studies (CA209063 and ONO-453802), and 3 phase 3 studies (CheckMate 017, CheckMate 057, and ATTRACTION-2) (Supplemental Table 1). The protocol and all amendments were approved by the institutional review board or independent ethics committee for each study center. All patients were required to provide written informed consent before enrollment.

The studies incorporated in this analysis, including nivolumab monotherapy cohorts of patients with GC/GEJC from the CheckMate 032 and ATTRACTION-2 studies [13, 14], enabled assessment of nivolumab PK in GC/GEJC relative to NSCLC second line or subsequent lines of treatment $(2 \mathrm{~L}+)$ in both Asian and non-Asian patients. Although nine patients with esophageal cancer (EC) were also included in the CheckMate 032 cohort, we will refer to these cohorts as GC/GEJC throughout the manuscript.

Serum nivolumab concentrations were analyzed using one of the three different validated bioanalytical assays: two different ligand-binding enzyme-linked immunosorbent assays (ELISAs) and an electrochemiluminescence (ECL) assay. The lower limits of quantification (LLOQ) were 1.2 or $1.0 \mu \mathrm{g} / \mathrm{mL}$ for ELISA assay and $0.2 \mu \mathrm{g} / \mathrm{mL}$ for ECL assay.

Nivolumab PK data were analyzed by nonlinear mixed effects modeling with NONMEM version 7.3 (ICON Development Solutions, Hanover, MD, USA) using the first-order 
conditional estimation method with interaction (FOCEI) and run using PsN (Version 4.4.8). Graphs were prepared using $\mathrm{R}$ software (Version 3.1.2).

\section{PopPK model}

The PopPK model was developed in three steps: base, full, and final models. The previously determined base structural model was a two-compartment, zero-order intravenous (IV) infusion and time-varying CL (sigmoidal- $E_{\max }$ function) with a proportional residual error model, with random effect on CL, central volume of distribution (VC), volume of distribution of peripheral compartment (VP), and $E_{\max }$, and correlation of random effect between CL and VC $[16,21]$. Based on previous findings, the following were included in the base model as covariates: BWT, eGFR, PS (derived from the Eastern Cooperative Oncology Group score), sex, race, and tumor type (GC/GEJC, or other cancer) on CL, and baseline BWT and sex on VC.

The full model included the assessment of the following pre-specified covariate effects on CL: baseline albumin, lactate dehydrogenase (LDH), tumor size, gastrectomy, and tumor type on $E_{\max }$. Prior gastrectomy was only assessed in patients with GC/GEJC. The proportion of patients with missing values for prior gastrectomy were different between CheckMate 032 and ATTRACTION-2 (22\% vs 3.3\%, respectively). The data for gastrectomy were not considered missing at random. Therefore, the missing values for gastrectomy were treated as "missing" without imputation to enable robust assessment of the effect of gastrectomy.

The final model was developed from the full model by backward elimination based on Bayesian information criterion (BIC). With this evaluation, the final model had the lowest BIC value. Baseline albumin [22, 23], LDH [16], tumor size [22], and gastrectomy [19, 20] have been identified as significant covariates on PK for antibodies against PD-1 (nivolumab and pembrolizumab), anti-PD-1 ligand (durvalumab), or other monoclonal antibodies targeting human epidermal growth factor receptor 2 (trastuzumab) or vascular endothelial growth factor A (bevacizumab), and were retained in the final model.

The final model was evaluated using prediction-corrected visual predictive check (pcVPC) [24], which provides a graphic assessment of the agreement between the time course of observed concentrations and those predicted by the model. The pcVPC was performed for patients with GC/ GEJC, as the purpose of the analysis was to describe the PK in this patient population. The pcVPC was used to compare the median, and 5th and 95th percentiles of the observed concentration-time data of patients in each group, with the $90 \%$ prediction interval of the corresponding statistics of simulated values. The simulated values were obtained from 1000 simulations of the model being evaluated (final model).

\section{Model application}

The maximum a posteriori (MAP) Bayesian parameter estimates from the final model were used to predict the summary measures of exposures for each patient in the analysis data set and for graphical assessment of ethnicity of patients with these exposures. In addition, nivolumab exposures for patients receiving the flat $240 \mathrm{mg}$ Q2W dosage regimen were predicted and compared with the weight-based $3 \mathrm{mg} /$ $\mathrm{kg}$ Q2W dosage regimen in patients with GC/GEJC.

\section{Results}

The analysis population consisted of 1302 patients with various recurrent/metastatic solid tumors (colorectal cancer, melanoma, castration-resistant prostate cancer, squamous and non-squamous NSCLC, and RCC), and included 387 patients with GC/GEJC for whom nivolumab serum concentration data were available (58 patients from CheckMate 032, and 329 patients from ATTRACTION-2). A total of 8585 observations of nivolumab serum concentrations were included in the PopPK analysis.

The diagnostic plots of observed and predicted nivolumab concentrations showed no indication of model bias over a wide range of concentrations (Supplemental Fig. 1). There were no marked trends in the conditional weighted residuals vs time (after first dose) diagnostic plot by tumor types (Supplemental Fig. 2), suggesting that the base model provides an adequate description of nivolumab PK for each tumor type.

The population mean CL among patients with GC/GEJC was 33\% higher, relative to that in patients with NSCLC $2 \mathrm{~L}+$ (Fig. 1). Over time, the population mean $\mathrm{CL}$ in patients with GC/GEJC decreased by $20 \%$ from baseline compared with $\sim 27 \%$ in patients with NSCLC $2 \mathrm{~L}+$ or other tumor types. The effect of GC/GEJC tumor type on $E_{\max }$ was lower compared with the reference tumor type (NSCLC 2L+). However, the CI was wide and included 1 , which suggested that this difference may not have clinical relevance.

The impact of covariate effect is presented in Fig. 1. Nivolumab CL and VC were higher in patients with higher BW. Nivolumab CL was higher in patients with lower baseline albumin. Race, sex, PS, baseline tumor size, baseline $\mathrm{LDH}$, and baseline eGFR were also not clinically relevant predictors of nivolumab CL $(<20 \%$ effect). The CL in patients with GC/GEJC who had prior gastrectomy was $18 \%$ lower than that in patients with GC/GEJC without gastrectomy. The estimates of covariate effects (and bootstrap 95\% CI) in full model are relative to CL, VC, or $E_{\max }$ at the reference values of the covariates (Fig. 1).

The final model was developed by backward elimination of the covariates in the full PopPK model, based on BIC. The final PopPK model contained the following 


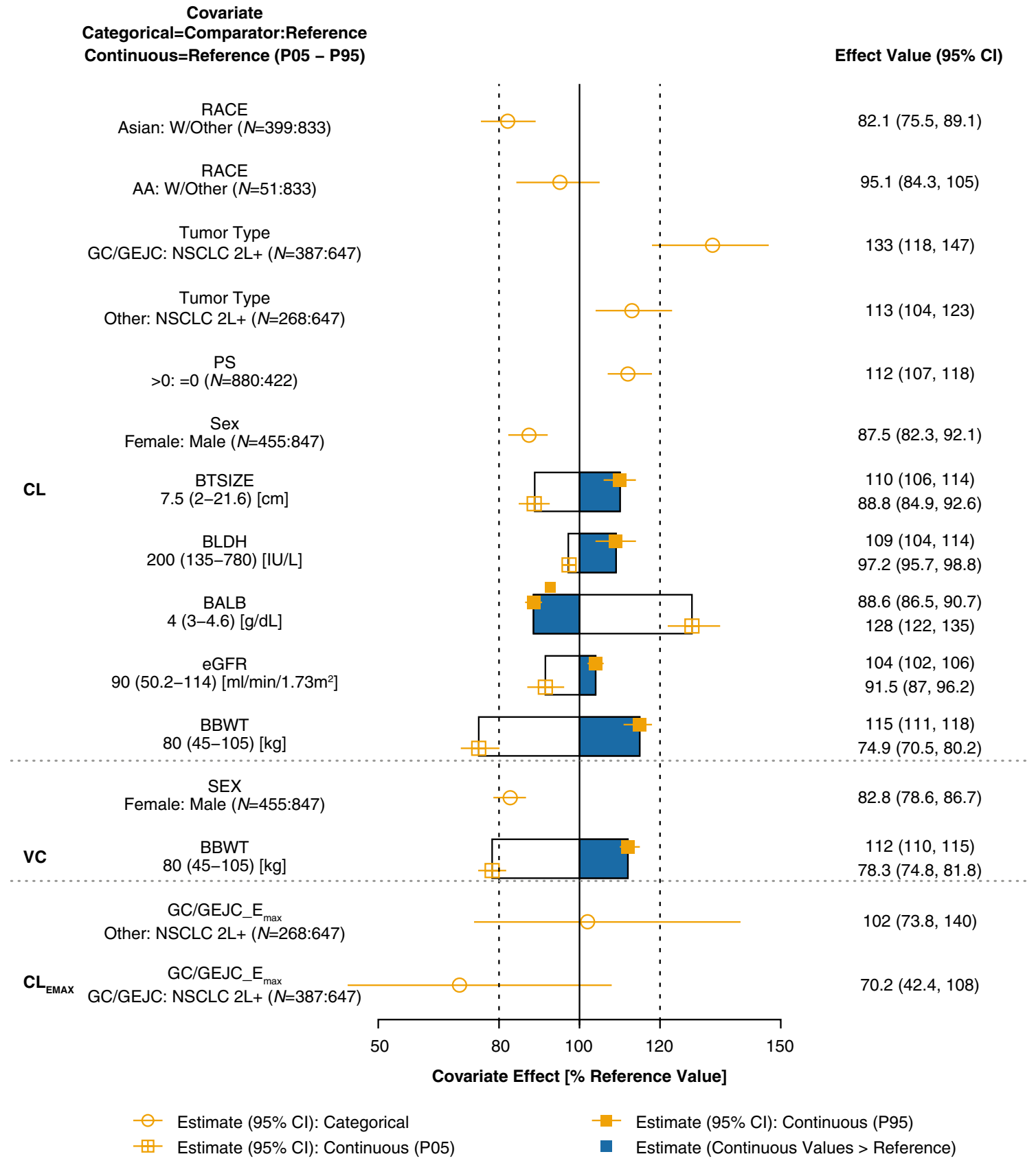

Fig. 1 Covariate effects on PK model parameters in the full PopPK model. Categorical covariate effects $(95 \% \mathrm{CI})$ are represented by open symbols (horizontal red lines). Continuous covariate effects $(95 \% \mathrm{CI})$ at the 5 th/95th percentiles of the covariate are represented by the end of horizontal boxes (horizontal red lines). Open/blue area of boxes represents the range of covariate effects from the median to the 5th/95th percentiles of the covariate. Reference patient was white/ other race male, $\mathrm{PS}=0, \mathrm{eGFR}=90 \mathrm{~mL} / \mathrm{min} / 1.73 \mathrm{~m}^{2}, \mathrm{BW}=80 \mathrm{~kg}$, NSCLC 2L+ tumor type. Parameter estimate in reference patient was

covariates: baseline BW, eGFR, sex, race, PS, baseline albumin, baseline LDH, prior gastrectomy, baseline tumor size, and tumor type (GC/GEJC or other cancer) on CL, and baseline BW and sex on VC (Table 1). considered to be $100 \%$ (vertical solid line), and dashed vertical lines are at $80 \%$ and $120 \%$ of this value. $A A$ African American race, $B A L B$ baseline albumin, $B L D H$ baseline lactate dehydrogenase, BTSIZE baseline tumor size, $B B W T$ baseline body weight, $C I$ confidence interval, $C L$ total body clearance, $e G F R$ baseline estimated glomerular filtration rate, $E_{\max }$ sigmoid-maximum effect, $G C$ gastric cancer, $G E J C$ gastro-esophageal junction cancer, $P S$ performance status, $V C$ volume of the central compartment, $W$ white

Results from the pcVPC assessment of predictive performance of the final PopPK model in patients with GC/GEJC are presented in Fig. 2. The pcVPC with (a) all available concentrations vs time after the previous dose and (b) only 
Table 1 PopPK model parameter estimates (final model)

\begin{tabular}{|c|c|c|c|}
\hline Name $^{\mathrm{a}}$ (units) & Estimate $^{\mathrm{b}}$ & Standard error $(\mathrm{RSE} \%)^{\mathrm{c}}$ & $95 \%$ Confidence interval ${ }^{\mathrm{d}}$ \\
\hline \multicolumn{4}{|l|}{ Fixed effects } \\
\hline $\mathrm{CL}(\mathrm{L} / \mathrm{h})$ & 0.011 & $0.000(4)$ & 0.010 to 0.012 \\
\hline $\mathrm{VC}(\mathrm{L})$ & 4.460 & $0.056(1)$ & 4.350 to 4.570 \\
\hline$Q(\mathrm{~L} / \mathrm{h})$ & 0.026 & $0.003(10)$ & 0.021 to 0.035 \\
\hline $\mathrm{VP}(\mathrm{L})$ & 2.520 & $0.119(5)$ & 2.270 to 2.790 \\
\hline CL_BBWT & 0.498 & $0.057(12)$ & 0.374 to 0.604 \\
\hline CL_GFR & 0.151 & $0.043(29)$ & 0.065 to 0.239 \\
\hline CL_SEX & -0.134 & $0.028(21)$ & -0.197 to -0.086 \\
\hline CL_PS & 0.117 & $0.024(20)$ & 0.065 to 0.166 \\
\hline CL_OTH & 0.128 & $0.035(28)$ & 0.050 to 0.195 \\
\hline CL_GC & 0.310 & 0.049 (16) & 0.201 to 0.399 \\
\hline CL_RAAA & -0.049 & $0.051(105)$ & -0.168 to 0.054 \\
\hline CL_RAAS & -0.201 & $0.040(20)$ & -0.279 to -0.120 \\
\hline VC_BBWT & 0.428 & $0.040(9)$ & 0.351 to 0.507 \\
\hline VC_SEX & -0.189 & $0.024(13)$ & -0.240 to -0.141 \\
\hline CL_EMAX & -0.285 & $0.051(18)$ & -0.408 to -0.177 \\
\hline CL_T50 & 1510 & $251(17)$ & 1040 to 2180 \\
\hline CL_HILL & 2.020 & $0.624(31)$ & 1.300 to 5.240 \\
\hline CL_BALB & -0.869 & $0.088(10)$ & -1.040 to -0.698 \\
\hline CL_BLDH & 0.379 & $0.106(28)$ & 0.165 to 0.577 \\
\hline CL_BTSIZE & 0.089 & 0.017 (19) & 0.057 to 0.122 \\
\hline CL_CASG & -0.193 & $0.040(21)$ & -0.281 to -0.110 \\
\hline CL_CASG_MIS & -0.112 & $0.089(80)$ & -0.278 to 0.066 \\
\hline \multicolumn{4}{|l|}{ Random effects } \\
\hline ZCL (-) & $0.094(0.307)$ & $0.009(9)$ & 0.077 to 0.114 \\
\hline $\mathrm{ZVC}(-)$ & $0.108(0.329)$ & $0.016(15)$ & 0.080 to 0.141 \\
\hline $\mathrm{ZVP}(-)$ & $0.314(0.560)$ & $0.042(14)$ & 0.237 to 0.422 \\
\hline ZEMAX (h) & $0.118(0.344)$ & $0.037(31)$ & 0.061 to 0.213 \\
\hline ZCL:ZVC (-) & $0.039(0.382)$ & $0.007(18)$ & 0.024 to 0.053 \\
\hline \multicolumn{4}{|l|}{ Residual error } \\
\hline $\operatorname{PERR}(-)$ & 0.219 & $0.009(4)$ & 0.202 to 0.239 \\
\hline
\end{tabular}

CL_T50 and CL_HILL govern the time-varying CL. The variance of random parameters on CL, VC, VP, $E_{\max }$, etc, is denoted as ZCL, ZVC, ZVP, and ZEMAX

$B A L B$ baseline albumin, $B L D H$ baseline lactate dehydrogenase, BTSIZE baseline tumor size, BBWT baseline body weight, $C A S G$ with prior gastrectomy, $C L$ total body clearance, $e G F R$ baseline estimated glomerular filtration rate, $G C$ gastric cancer, $M I S$, missing, $O T H$ other tumor types, $P E R R$ proportional error, $P S$ performance status, $P S N$ Perl-speaks-NONMEM, $Q$ inter-compartmental clearance, RAAA African American race, $R A A S$ Asian race, $V C$ volume of the central compartment, $V P$ volume of distribution of the peripheral compartment

${ }^{a}$ Random effects and residual error parameter names containing a colon (:) denote correlated parameters

${ }^{\mathrm{b}}$ Random effects and residual error parameter estimates are shown as variance (standard deviation) for diagonal elements $\left(\omega_{i, i}\right.$ or $\left.\sigma_{i, i}\right)$ and covariance (correlation) for off-diagonal elements $\left(\omega_{i, j}\right.$ or $\left.\sigma_{i, j}\right)$

${ }^{\mathrm{c}} \mathrm{RSE} \%$ is the relative standard error (standard error as a percentage of estimate)

${ }^{\mathrm{d}}$ Confidence intervals of random effects and residual error parameters are for variance or covariance trough concentrations vs time after the first dose demonstrate that the model adequately characterized the trend of the concentration-time profile. The median observed profile tracked well with the simulation results and the extent of variability based on the simulation was similar to the extent of variability in the observed data. In addition, there was reasonably good agreement between the 5th and 95th percentiles of the observed and simulated data.

The final PopPK model was used to assess the effect of race (Asian vs non-Asian) on nivolumab exposure. This analysis included 328 Asian patients and 59 non-Asian patients (1 patient from the ATTRACTION-2 study was 


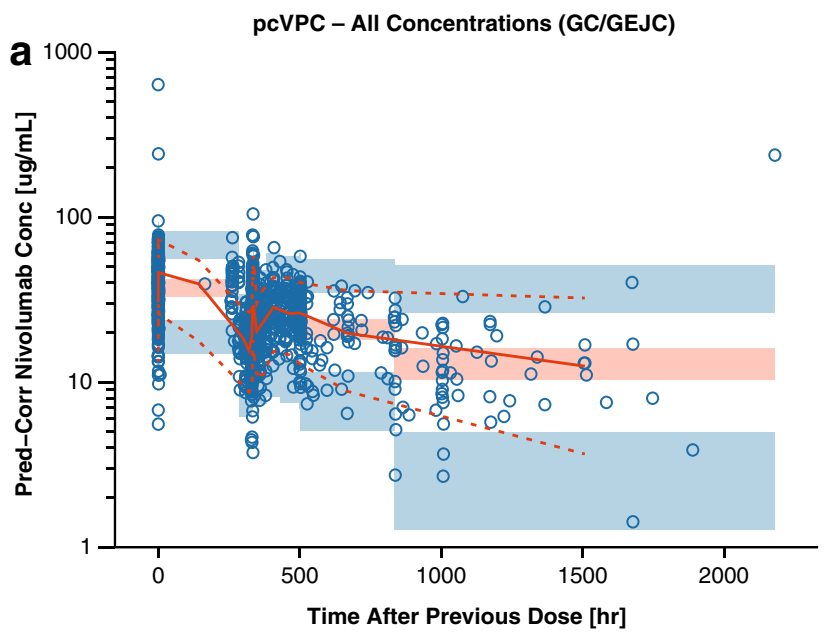

pcVPC - Trough Concentrations (GC/GEJC)

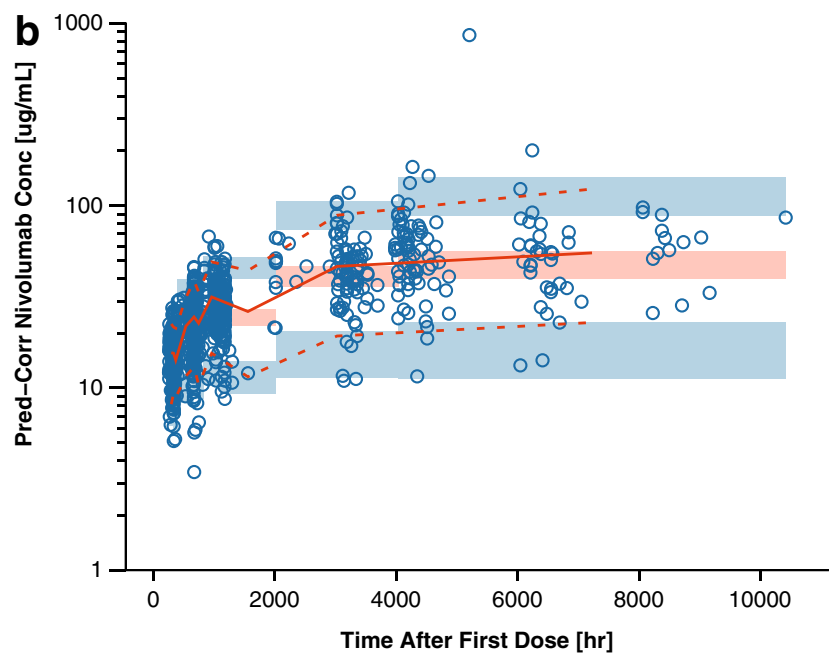

Fig. 2 Prediction-corrected visual predictive check (pcVPC) of the final PopPK model in patients with GC/GEJC in a all concentrations vs actual time after previous dose and $\mathbf{b}$ trough concentrations vs actual time after first dose. Blue circles are observed data. The red lines represent the 5th, 50th, and 95th percentiles of observed data. The shaded areas represent the simulation-based $95 \%$ confidence intervals for the 5th, 50th, and 95th percentiles of the predicted data. Conc concentration, GC gastric cancer, GEJC gastro-esophageal junction cancer, Pred-Corr prediction corrected

a Native Hawaiian and was categorized as non-Asian). Asian and non-Asian patients with GC/GEJC had similar nivolumab exposure measurements following $3 \mathrm{mg} / \mathrm{kg}$ dosing. Geometric means (CV\%) in Asian vs non-Asian patients were as follows: trough serum concentration following dose $1\left(C_{\min 1} ; 13.3\right.$ [26\%] vs 14.5 [25\%], respectively), peak serum concentration following dose $1\left(C_{\max 1}\right.$; 42.4 [45\%] vs 49.4 [32\%], respectively), average serum concentration following dose $1\left(C_{\text {avg } 1} ; 20.6\right.$ [20\%] vs 23.7 [20\%], respectively), trough serum concentration at steady state $\left(C_{\text {minss }} ; 33.4[40 \%]\right.$ vs 32.5 [38\%], respectively), peak
Nivolumab $\mathrm{C}_{\text {avg1 }}$ Estimates in GC/GEJC Population

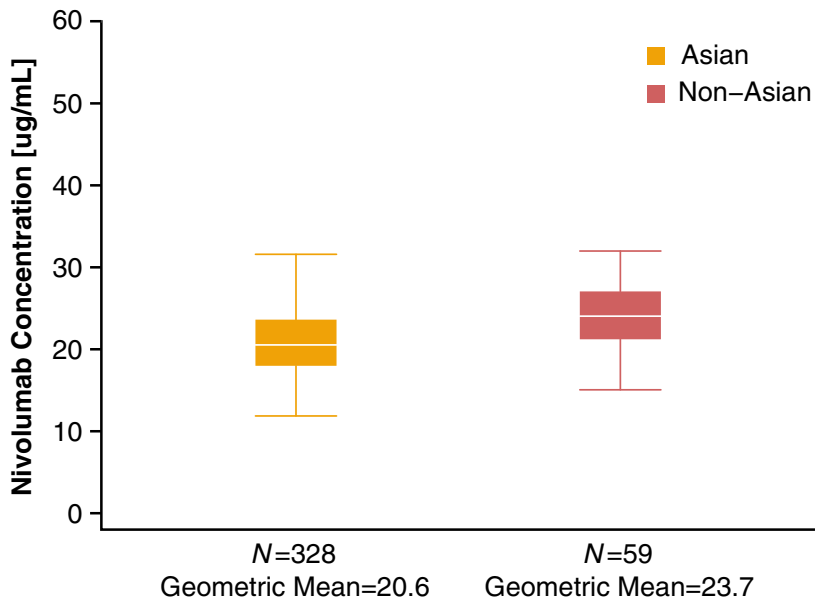

Fig. 3 Distribution of nivolumab $C_{\mathrm{avg} 1}$ estimates in Asian and nonAsian patients with GC/GEJC (3 mg/kg Q2W dose). The boxes represent the 25th, 50th, and 75th percentiles of the distribution. The whiskers extend to 1.5 times the interquartile range. This analysis included 328 Asian patients and 59 non-Asian patients (1 patient from the ATTRACTION-2 study was a Native Hawaiian and was categorized as non-Asian). $C_{a v g l}$ average serum concentration following dose 1, GC gastric cancer, GEJC gastro-esophageal junction cancer, $Q 2 W$ every 2 weeks

serum concentration at steady state $\left(C_{\text {maxss }} ; 77.4[32 \%]\right.$ vs 82.7 [30\%], respectively), and average serum concentration at steady state $\left(C_{\text {avgss }} ; 47.8\right.$ [32\%] vs 49.1 [31\%], respectively) (Fig. 3; Supplemental Table 2). Nivolumab exposure after the first dose was slightly higher in non-Asian patients with GC/GEJC (geometric mean $C_{\text {avg } 1}=23.7$ ) compared to Asian patients with GC/GEJC (geometric mean $C_{\text {avg1 }}=20.6$; Supplemental Table 2), but the distribution overlapped (Fig. 3), which indicates that race was not a clinically relevant covariate.

The final PopPK model was also used to compare nivolumab exposures following a flat $240 \mathrm{mg}$ Q2W dose regimen with a weight-based $3 \mathrm{mg} / \mathrm{kg}$ Q2W dose regimen in patients with GC/GEJC both in the overall population and in Asian and non-Asian subpopulations (Fig. 4a-c; Table 2). In the overall GC/GEJC patient population, nivolumab exposure following the $240 \mathrm{mg}$ Q2W regimen was predicted to be $\sim 40 \%$ higher compared with exposure following a $3 \mathrm{mg} /$ kg Q2W regimen (Table 2). In Asian patients, nivolumab exposure after administration of the $240 \mathrm{mg}$ Q2W dose (median $\mathrm{C}_{\text {avg1 }}$ [P05-P95]) was predicted to be $31.0 \mu \mathrm{g} / \mathrm{mL}$ (21.0-41.5), 46\% higher than exposure achieved following administration of nivolumab $3 \mathrm{mg} / \mathrm{kg}$ Q2W (Table 2). However, exposures among non-Asian patients with $\mathrm{GC} /$ GEJC were predicted to be similar following administration of either of the two regimens, with the difference between the geometric means across all exposure metrics for the two regimens predicted to be $<10 \%$ (Table 2). 
Fig. 4 Model-predicted $\mathrm{C}_{\text {avgl }}$ following nivolumab $240 \mathrm{mg}$ Q2W versus $3 \mathrm{mg} / \mathrm{kg}$ Q2W in the a overall GC/GEJC population, b non-Asian GC/GEJC population, and $\mathbf{c}$ Asian GC/GEJC population. The boxes represent the 25th, 50th, and 75th percentiles of the distribution. The whiskers extend to 1.5 times the interquartile range. This analysis included 328 Asian patients and 59 non-Asian patients (1 patient from the ATTRACTION-2 study was a Native Hawaiian and was categorized as non-Asian). $C_{a v g l}$ average serum concentration following dose 1, GC gastric cancer, GEJC gastro-esophageal junction cancer, $Q 2 W$ every 2 weeks

\section{Discussion}

In the present analyses, nivolumab concentration data from patients with GC/GEJC, NSCLC 2L+, and other solid tumor types were described by a linear, 2-compartment, zeroorder input IV infusion model with first-order elimination and time-varying CL, which was the same as a previously developed model, except for the incorporation of additional covariate-parameter effects [16]. The full model approach was used for covariate assessment, as it provides an unbiased estimate of covariate effects, and the final model was used for prediction of nivolumab exposures. Based on the results of pcVPC, the PopPK model provided an adequate description of nivolumab concentration-time data in patients with GC/GEJC. The model was deemed appropriate to predict and compare exposures in Asian and non-Asian patients with GC/GEJC, and to predict and compare exposures with flat (240 mg Q2W) and weight-based (3 mg/kg Q2W) nivolumab dosing in patients with GC/GEJC. The effects of other covariates on CL that were previously evaluated (e.g., PS, eGFR, and BW [16]) remained in the current model with similar point estimates, illustrating that the addition of data from patients with GC/GEJC did not have a large impact on these effects. Furthermore, as previously reported [16], it was not expected that PS, eGFR, baseline LDH, or baseline tumor size would be clinically relevant in prediction of nivolumab CL, because the magnitudes of difference were $<20 \%$. The additional covariate-parameter relationships included in the present analysis were the effect of gastrectomy on CL and the effect of tumor type on $E_{\max }$.

The main covariate of interest in the current analyses was GC/GEJC tumor type. Patients with GC/GEJC had $\sim 33 \%$ greater baseline CL relative to that of patients with NSCLC 2L+. Interestingly, the magnitude of the effect of $\mathrm{GC}$ tumor type was greater than that observed in a previous nivolumab PopPK analysis relative to the NSCLC $2 \mathrm{~L}+$ reference $(\sim 20 \%)$. However, the previous estimate was based on data from 58 patients with GC/GEJC from the CheckMate 032 study [14]. In the current analyses, 329 patients with GC/GEJC from the ATTRACTION-2 study [13] were added, for a total of 387 patients with GC/GEJC, enabling a more robust assessment of GC/GEJC tumor type effect. In addition to the tumor type, another difference between
Overall GC/GEJC Population

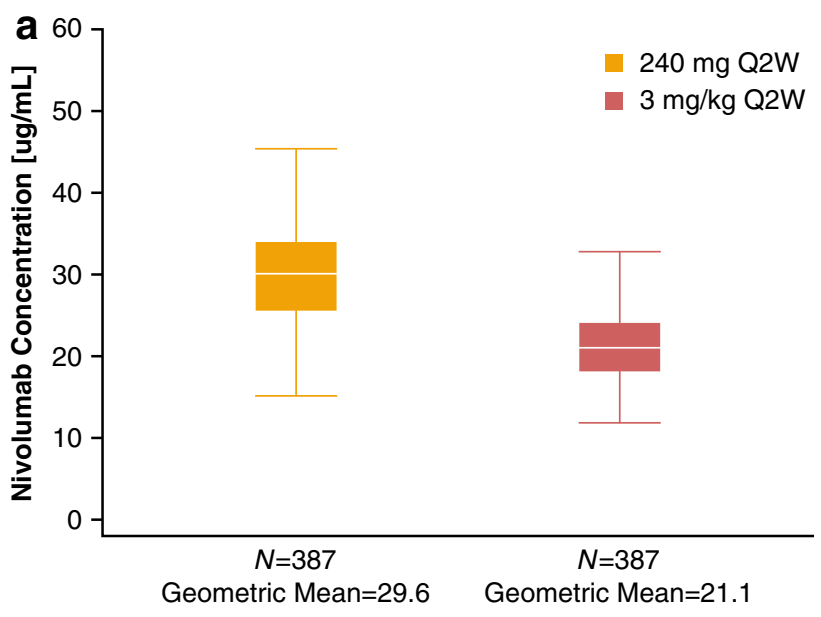

Non-Asian GC/GEJC Population

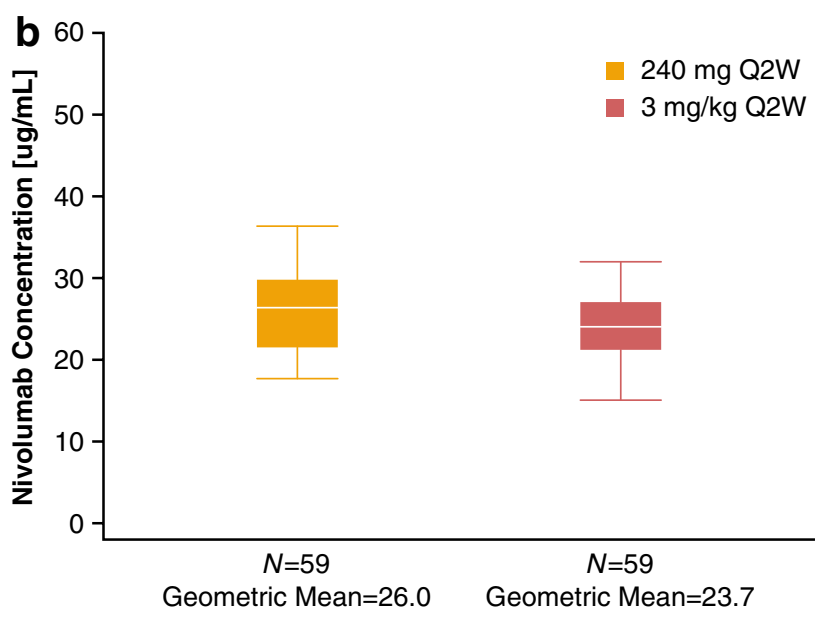

Asian GC/GEJC Population

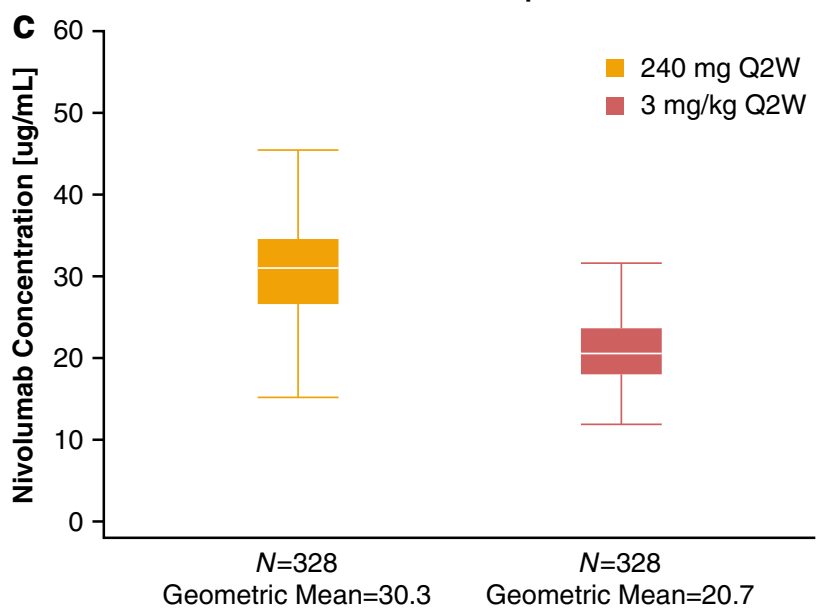

this analysis and the previous PopPK analysis was that the majority of patients included in the current analysis were Asian. The results of the current PopPK analyses were 
Table 2 Nivolumab exposure following $240 \mathrm{mg}$ Q2W or $3 \mathrm{mg} / \mathrm{kg}$ Q2W dosage regimens in patients with GC/GEJC

\begin{tabular}{|c|c|c|c|c|c|c|}
\hline \multirow[t]{2}{*}{ Population } & \multirow{2}{*}{$\begin{array}{l}\text { Exposure parameter } \\
(\mu \mathrm{g} / \mathrm{mL})\end{array}$} & \multicolumn{2}{|c|}{ Geometric mean (CV\%) } & \multirow{2}{*}{$\begin{array}{l}\text { GM diff } \\
\text { percent }^{\mathrm{a}}(\%)\end{array}$} & \multicolumn{2}{|l|}{ Median (P05-P95) ${ }^{\mathrm{b}}$} \\
\hline & & $240 \mathrm{mg}$ Q2W & $3 \mathrm{mg} / \mathrm{kg}$ Q2W & & $240 \mathrm{mg}$ Q2W & $3 \mathrm{mg} / \mathrm{kg}$ Q2W \\
\hline \multirow[t]{6}{*}{ Overall } & $C_{\min 1}$ & $18.9(28.4)$ & $13.5(26.1)$ & 40.0 & $19.8(11.3-28.1)$ & $13.5(8.17-20.4)$ \\
\hline & $C_{\max 1}$ & $61.0(51.8)$ & $43.4(43.1)$ & 40.6 & $60.6(39.4-92.9)$ & $43.2(29.4-61.0)$ \\
\hline & $C_{\text {avg1 }}$ & $29.6(21.7)$ & $21.1(20.3)$ & 40.3 & $30.1(20.1-40.9)$ & $21.1(15.4-29.3)$ \\
\hline & $C_{\text {minss }}$ & $46.7(43.3)$ & $33.3(39.7)$ & 40.2 & $48.3(21.8-86.7)$ & $34.3(15.8-60.9)$ \\
\hline & $C_{\text {maxss }}$ & $110.0(38.5)$ & $78.2(32.1)$ & 40.7 & $112.0(66.8-169)$ & $78.4(53.6-114.0)$ \\
\hline & $C_{\text {avgss }}$ & $67.4(35.3)$ & $48.0(32.0)$ & 40.4 & $68.8(37.0-113)$ & $48.0(27.2-79.7)$ \\
\hline \multirow[t]{6}{*}{ Non-Asian } & $C_{\min 1}$ & $15.8(28.5)$ & $14.5(25.4)$ & 9.0 & $15.9(10.0-24.4)$ & $14.7(8.9-21.9)$ \\
\hline & $C_{\max 1}$ & $54.2(40.7)$ & $49.4(32.2)$ & 9.7 & $53.4(35.7-77.1)$ & $49.5(34.5-67.0)$ \\
\hline & $C_{\text {avg1 }}$ & $26.0(23.6)$ & $23.7(19.9)$ & 9.7 & $26.4(18.4-35.7)$ & $24.0(16.4-30.8)$ \\
\hline & $C_{\text {minss }}$ & $35.6(40.3)$ & $32.5(38.3)$ & 9.5 & $35.6(19.1-68.1)$ & $32.7(18.0-57.1)$ \\
\hline & $C_{\text {maxss }}$ & $90.6(35.9)$ & $82.7(29.5)$ & 9.6 & $86.4(58.6-134)$ & $82.7(54.8-115.0)$ \\
\hline & $C_{\text {avgss }}$ & $53.8(33.4)$ & $49.1(30.8)$ & 9.6 & $53.5(31.5-90.8)$ & $48.8(29.7-76.0)$ \\
\hline \multirow[t]{6}{*}{ Asian } & $C_{\min 1}$ & $19.5(27.3)$ & $13.3(26.0)$ & 46.6 & $20.3(11.7-28.9)$ & $13.4(7.98-20.1)$ \\
\hline & $C_{\max 1}$ & $62.3(52.8)$ & $42.4(44.9)$ & 46.9 & $62.0(39.6-94.4)$ & $42.4(29.1-60.7)$ \\
\hline & $C_{\text {avg1 }}$ & $30.3(20.7)$ & $20.7(19.6)$ & 46.4 & $31.0(21.0-41.5)$ & $20.5(15.3-28.8)$ \\
\hline & $C_{\text {minss }}$ & $49.1(41.9)$ & $33.4(39.9)$ & 47.0 & $51.0(23.0-89.5)$ & 34.7 (15.6-61.6) \\
\hline & $C_{\text {maxss }}$ & $114.0(38.0)$ & $77.4(32.5)$ & 47.3 & $115.0(70.3-171)$ & $77.3(53.6-114.0)$ \\
\hline & $C_{\text {avgss }}$ & $70.2(34.2)$ & $47.8(32.2)$ & 46.9 & $72.0(40.1-115)$ & $47.9(27.0-79.6)$ \\
\hline
\end{tabular}

$C_{\text {avgl }}$ average serum concentration following dose $1, C_{\text {avgss }}$ average serum concentration at steady state, $C_{\operatorname{maxl}}$ peak serum concentration following dose $1, C_{\text {maxss }}$ peak serum concentration at steady state, $C_{\min l}$ trough serum concentration following dose $1, C_{\text {minss }}$ trough serum concentration at steady state, $C V$ coefficient of variation; $G M$ geometric mean, $Q 2 W$ every 2 weeks

${ }^{\mathrm{a}} \mathrm{GM}$ diff percent $=[($ geometric mean of $240 \mathrm{mg}$ Q2W - geometric mean of $3 \mathrm{mg} / \mathrm{kg} \mathrm{Q} 2 \mathrm{~W}) /$ geometric mean of $3 \mathrm{mg} / \mathrm{kg}$ Q2W $] \times 100$

${ }^{\mathrm{b}} \mathrm{P} 05$ : the 5th percentile; P95: the 95th percentile

consistent with those of bevacizumab and trastuzumab, in which CL was greater in patients with GC when compared with other solid tumor types $[19,20]$. The present study also evaluated the effect of GC/GEJC on the change in $\mathrm{CL}\left(E_{\max }\right)$ and found that the effect of GC/GEJC on $E_{\max }$ was lower than that of NSCLC $2 \mathrm{~L}+$. However, the effect of GC/GEJC on $E_{\max }$ was removed from the model in the backward elimination process, indicating that it was not a significant covariate.

For patients with GC/GEJC, nivolumab exposure was lower relative to the reference NSCLC $2 \mathrm{~L}+$ patient group, as a result of the higher CL in the GC/GEJC population. However, results of the ATTRACTION-2 study demonstrated that treatment with nivolumab $3 \mathrm{mg} / \mathrm{kg}$ Q2W resulted in superior survival benefit compared with placebo and a manageable safety profile in patients with unresectable or advanced GC/GEJC previously treated with $\geq 2$ lines of prior chemotherapy [13], suggesting that this dosing regimen was beneficial in this population, despite the lower exposures vs NSCLC. In addition, flat exposure-response relationships for nivolumab efficacy across dose ranges that include $3 \mathrm{mg} / \mathrm{kg}$ Q2W have been demonstrated in melanoma [25], suggesting that lower exposure in patients with GC/ GEJC is not expected to reduce the efficacy of nivolumab.
The effect of race was evaluated in the full model, and the exposures between Asian and non-Asian patients were compared using data from the ATTRACTION-2 and CheckMate 032 studies [13, 14]. The effect of Asian vs non-Asian race covariate indicated that CL in Asian patients was 18\% lower than in non-Asian patients. However, as the exposure ranges overlapped between these patient populations, the effect of race was not considered clinically relevant.

Because there were more Asian than non-Asian patients included in the analysis (328 Asian GC/GEJC patients and 59 non-Asian GC/GEJC patients), it is possible that the effects of tumor type and Asian race could have been confounded. Therefore, it is important to consider these results in the context of the previous analyses. As described above, the effect of GC/GEJC tumor type was greater than the previous results when only data from non-Asian patients were used to estimate the effect of GC/GEJC tumor type. The effect of Asian race on CL in the current analyses is numerically lower than the previously reported estimate. If Asian race is confounded with the tumor type effect, the estimates for GC/GEJC would be expected to be lower than the estimates achieved using data from only non-Asian patients with GC/GEJC, which was not the case. Similarly, if the effects of tumor type confounded the effects of Asian race, 
it would be expected that the effect of Asian race would have been smaller, or closer to unity. While this does not eliminate the possibility that the estimates of the effects of GC/GEJC tumor type and Asian race on nivolumab CL are confounded, the increased magnitudes of effects of both GC/ GEJC tumor type and Asian race in the current analyses vs previous estimates suggest that enriching for Asian patients with GC/GEJC in the current analyses did not confound the estimates.

Patients with GC/GEJC who had prior gastrectomy had $18 \%$ lower CL compared with patients with GC/GEJC without gastrectomy. These findings were consistent with previously reported analyses of other monoclonal antibodies [19, 20]. Currently, the reasons for this finding are unknown, but it has been postulated that patients who have had gastrectomy have better clinical outcomes and health status compared with those who have not had gastrectomy, and healthier patients with GC have slower CL of other monoclonal antibodies such as bevacizumab [20]. Results from the ATTRACTION-2 study of nivolumab vs placebo in Asian patients with GC/GEJC indicated that nivolumab provided an OS benefit regardless of prior gastrectomy, although the reduction of risk of death was numerically greater in patients with gastrectomy [13].

The present analysis showed that the nivolumab CL in patients with GC/GEJC decreased over time, consistent with what has previously been observed with other tumor types [16]. The reason for this finding is unknown, but it could be explained by the relative health of those individuals and/or the response to nivolumab treatment. A previous study demonstrated that improving disease status of patients treated with nivolumab can be associated with decreasing CL leading to increased nivolumab exposure [26].

When the model was applied to predict exposures at the $240 \mathrm{mg}$ Q2W flat dose of nivolumab (regardless of tumor effect on CL), the exposures were predicted to be comparable to those following administration of $3 \mathrm{mg} / \mathrm{kg}$ Q2W nivolumab in non-Asian patients with GC/GEJC $(<10 \%$ difference). However, the predicted nivolumab exposures following the $240 \mathrm{mg}$ Q2W regimen were greater ( 46\%) than those achieved with the $3 \mathrm{mg} / \mathrm{kg}$ Q2W regimen in the Asian GC/GEJC population.

Among all patients with GC/GEJC included in the analysis, the exposure estimates following treatment with nivolumab $240 \mathrm{mg}$ Q2W were $~ 40 \%$ higher compared with exposure following nivolumab $3 \mathrm{mg} / \mathrm{kg}$ Q2W. As described earlier, the majority of patients included in the overall $\mathrm{GC} /$ GEJC population were Asian (329 out of 387 total patients). It is possible that the different results between the overall population and non-Asian population could be explained by the fact that median BW among Asian patients was lower than the $80 \mathrm{~kg}$ reference used to calculate the nivolumab $240 \mathrm{mg}$ Q2W dose. However, the predicted $240 \mathrm{mg}$ Q2W exposure (median $C_{\text {avg1 }}$ ) of $31.0 \mu \mathrm{g} / \mathrm{mL}$ in Asian patients in this study was still below the previously reported exposure of $86.5 \mu \mathrm{g} / \mathrm{mL}$ achieved for a well-tolerated dose of $10 \mathrm{mg} / \mathrm{kg}$ Q2W [27]. Even the greater exposures remained within the range of nivolumab exposures that are well tolerated, providing an acceptable safety margin. Previous exposure-risk analyses have indicated that the safety risk does not increase with nivolumab doses up to $10 \mathrm{mg} / \mathrm{kg}$ Q2W $[17,25]$. In addition, these greater exposures using a flat dose regimen would not be expected to negatively affect efficacy, based on the results of a nivolumab benefit-risk profile analysis in patients with solid tumors [27]. Overall, in patients with GC/GEJC, exposures following nivolumab $240 \mathrm{mg}$ Q2W are predicted to provide similar responses and an acceptable safety profile to those following administration of nivolumab $3 \mathrm{mg} / \mathrm{kg}$ Q2W.

In summary, although patients with GC/GEJC had greater CL of nivolumab compared with patients with NSCLC, given the superior OS benefit observed in the ATT RACTION-2 study [13], lower exposure in patients with GC/GEJC did not result in reduced efficacy. Furthermore, nivolumab $C_{\text {avg1 }}$ was not significantly associated with efficacy over the range of nivolumab doses up to $10 \mathrm{mg} / \mathrm{kg}$ in patients with melanoma [25] and NSCLC [17]. Nivolumab exposures following $3 \mathrm{mg} / \mathrm{kg}$ Q2W dosage in the current analysis were similar in Asian and non-Asian patients with chemotherapy-refractory GC or GEJC, and both patient populations gained clinically meaningful and durable antitumor activity and manageable safety from nivolumab monotherapy in clinical trials $[13,14]$. Finally, exposures following nivolumab $240 \mathrm{mg}$ Q2W in non-Asian or Asian GC/GEJC patients were predicted to be similar or higher than those following $3 \mathrm{mg} / \mathrm{kg}$ Q2W, but even the highest predicted exposures were well within the acceptable safety margin [27]. This result indicates that $240 \mathrm{mg}$ Q2W would provide similar clinical activity and safety profiles as nivolumab $3 \mathrm{mg} / \mathrm{kg}$ Q2W in Asian and non-Asian patients with GC/GEJC and confirms that use of flat dose is an option in these patient populations.

Acknowledgements We thank the following colleagues at BristolMyers Squibb for their contributions to this work: Erin Dombrowsky for data management and Bradly Boone for nivolumab bioanalysis. The studies included in this PopPK analysis were funded by Bristol-Myers Squibb (Princeton, NJ, USA) and Ono Pharmaceutical Co., Ltd (Osaka, Japan). Professional medical writing and editorial assistance were provided by Ewa Wandzioch, Ph.D., and Christine Craig of PAREXEL International, funded by Bristol-Myers Squibb.

\section{Compliance with ethical standards}

Conflict of interest This study was supported by Bristol-Myers Squibb. M. Osawa and M. Hasegawa are employees of Bristol-Myers Squibb K.K, Tokyo, Japan; M. Osawa owns stock in the company. A. Roy and A. Bello are employees of Bristol-Myers Squibb, Princeton, NJ, 
USA; A. Roy owns stock in the company. M. W. Hruska owns stock and was employed by Bristol-Myers Squibb at the time this work was completed.

Data Sharing BMS policy on data sharing may be found at https:// www.bms.com/researchers-and-partners/independent-research/datasharing-request-process.html.

OpenAccess This article is distributed under the terms of the Creative Commons Attribution 4.0 International License (http://creativeco mmons.org/licenses/by/4.0/), which permits unrestricted use, distribution, and reproduction in any medium, provided you give appropriate credit to the original author(s) and the source, provide a link to the Creative Commons license, and indicate if changes were made.

\section{References}

1. Bray F, Ferlay J, Soerjomataram I, Siegel RL, Torre LA, Jemal A (2018) Global cancer statistics 2018: GLOBOCAN estimates of incidence and mortality worldwide for 36 cancers in 185 countries. CA Cancer J Clin 0:1-31

2. National Comprehensive Cancer Network (2018) Clinical practice guidelines in oncology gastric cancer. Version 2.2018. https ://www.nccn.org/professionals/physician_gls/pdf/gastric.pdf. Accessed 22 May 2018

3. National Comprehensive Cancer Network (2018) Clinical practice guidelines in oncology esophageal and esophagogastric junction cancer. Version 2.2018. https://www.nccn.org/professionals/physi cian_gls/pdf/esophageal.pdf. Accessed 22 May 2018

4. Ford HE, Marshall A, Bridgewater JA, Janowitz T, Coxon FY, Wadsley J, Mansoor W, Fyfe D, Madhusudan S, Middleton GW, Swinson D, Falk S, Chau I, Cunningham D, Kareclas P, Cook N, Blazeby JM, Dunn JA, Investigators C- (2014) Docetaxel versus active symptom control for refractory oesophagogastric adenocarcinoma (COUGAR-02): an open-label, phase 3 randomised controlled trial. Lancet Oncol 15:78-86

5. Kang JH, Lee SI, Lim DH, Park KW, Oh SY, Kwon HC, Hwang IG, Lee SC, Nam E, Shin DB, Lee J, Park JO, Park YS, Lim HY, Kang WK, Park SH (2012) Salvage chemotherapy for pretreated gastric cancer: a randomized phase III trial comparing chemotherapy plus best supportive care with best supportive care alone. J Clin Oncol 30:1513-1518

6. Wilke H, Muro K, Van Cutsem E, Oh SC, Bodoky G, Shimada $Y$ et al (2014) Ramucirumab plus paclitaxel versus placebo plus paclitaxel in patients with previously treated advanced gastric or gastro-oesophageal junction adenocarcinoma (RAINBOW): a double-blind, randomised phase 3 trial. Lancet Oncol 15:1224-1235

7. Wang C, Thudium KB, Han M, Wang XT, Huang H, Feingersh D, Garcia C, Wu Y, Kuhne M, Srinivasan M, Singh S, Wong S, Garner N, Leblanc H, Bunch RT, Blanset D, Selby MJ, Korman AJ (2014) In vitro characterization of the anti-PD-1 antibody nivolumab, BMS-936558, and in vivo toxicology in non-human primates. Cancer Immunol Res 2:846-856

8. (2018) Opdivo (nivolumab) injection for intravenous use. [Prescribing information]. Bristol-Myers Squibb Company, Princeton, NJ

9. Press release from Bristol-Myers Squibb (2017) Japan Ministry of Health, Labor and Welfare approves Opdivo (nivolumab) for the treatment of patients with unresectable advanced or recurrent gastric cancer which has progressed after chemotherapy. https:// news.bms.com/press-release/corporatefinancial-news/japan-minis try-health-labor-and-welfare-approves-opdivo-nivolu. Accessed 10 July 2018

10. Press release from Ono Pharmaceutical CO., LTD. (2018) Opdivo $^{\circledR}$ (Nivolumab) intravenous infusion approved for supplemental indication of advanced or recurrent gastric or gastroesophageal junction adenocarcinoma and for expanded use in recurrent or advanced classical hodgkin lymphoma in South Korea. https ://www.ono.co.jp/eng/news/pdf/sm_cn180326.pdf. Accessed 10 July 2018

11. Press release from Ono Pharmaceutical CO., LTD. (2018) Opdivov $^{\circledR}$ (Nivolumab) intravenous infusion approved in Taiwan for supplemental indication of advanced or recurrent gastric cancer or gastro-esophageal junction cancer. https://www. ono.co.jp/eng/news/pdf/sm_cn180123.pdf. Accessed 10 July 2018

12. Swissmedic (2018) Swiss summary of the risk management plan for nivolumab (OPDIVO ${ }^{\circledR}$ ) version 6. Bristol-Myers Squibb Research and Development, Pennington

13. Kang YK, Boku N, Satoh T, Ryu MH, Chao Y, Kato K et al (2017) Nivolumab in patients with advanced gastric or gastrooesophageal junction cancer refractory to, or intolerant of, at least two previous chemotherapy regimens (ONO-4538-12, ATT RACTION-2): a randomised, double-blind, placebo-controlled, phase 3 trial. Lancet 390:2461-2471

14. Janjigian YY, Bendell J, Calvo E, Kim JW, Ascierto PA, Sharma P, Ott PA, Peltola K, Jaeger D, Evans J, de Braud F, Chau I, Harbison CT, Dorange C, Tschaika M, Le DT (2018) CheckMate-032 study: efficacy and safety of nivolumab and nivolumab plus ipilimumab in patients with metastatic esophagogastric cancer. J Clin Oncol. https://doi.org/10.1200/ JCO.2017.76.6212

15. Chau I, Chen LT, Kang YK, Satoh T, Kato K, Chung HC, Kang WK, Chao Y, Chen JS, Ott PA, Le DT, Zhao H, Jimenez-Exposito MJ, Janjigian YY, Boku N (2018) Nivolumab safety profile in Asian and western patients with chemotherapy-refractory advanced gastric/gastroesophageal junction cancer from the ATT RACTION-2 and CheckMate-032 trials. Presented at the 2018 Gastrointestinal Cancers Symposium of the American Society of Clinical Oncology; January 18-20, 2018; San Francisco, CA, USA. Abstract 90

16. Bajaj G, Wang X, Agrawal S, Gupta M, Roy A, Feng Y (2017) Model-based population pharmacokinetic analysis of nivolumab in patients with solid tumors. CPT Pharmacomet Syst Pharmacol 6:58-66

17. Feng Y, Wang X, Bajaj G, Agrawal S, Bello A, Lestini B, Finckenstein FG, Park JS, Roy A (2017) Nivolumab exposure-response analyses of efficacy and safety in previously treated squamous or nonsquamous non-small cell lung cancer. Clin Cancer Res 23:5394-5405

18. Wang X, Feng Y, Bajaj G, Gupta M, Agrawal S, Yang A, Park JS, Lestini B, Roy A (2017) Quantitative characterization of the exposure-response relationship for cancer immunotherapy: a case study of nivolumab in patients with advanced melanoma. CPT Pharmacomet Syst Pharmacol 6:40-48

19. Cosson VF, Ng VW, Lehle M, Lum BL (2014) Population pharmacokinetics and exposure-response analyses of trastuzumab in patients with advanced gastric or gastroesophageal junction cancer. Cancer Chemother Pharmacol 73:737-747

20. Han K, Jin J, Maia M, Lowe J, Sersch MA, Allison DE (2014) Lower exposure and faster clearance of bevacizumab in gastric cancer and the impact of patient variables: analysis of individual data from AVAGAST phase III trial. AAPS J 16:1056-1063

21. Raybon J, Statkevich P, Feng Y, Zhu L, Hruska M, Bello A, Agrawal S, Roy A, Bajaj G (2017) Characterization of nivolumab pharmacokinetics incorporating time-varying clearance in cancer 
patients. In: American Society for clinical pharmacology and therapeutics annual meeting, Washington, DC, USA

22. Baverel PG, Dubois VFS, Jin CY, Zheng Y, Song X, Jin X, Mukhopadhyay P, Gupta A, Dennis PA, Ben Y, Vicini P, Roskos L, Narwal R (2018) Population pharmacokinetics of durvalumab in cancer patients and association with longitudinal biomarkers of disease status. Clin Pharmacol Ther 103:631-642

23. Ahamadi M, Freshwater T, Prohn M, Li CH, de Alwis DP, de Greef R, Elassaiss-Schaap J, Kondic A, Stone JA (2017) Modelbased characterization of the pharmacokinetics of pembrolizumab: a humanized anti-PD-1 monoclonal antibody in advanced solid tumors. CPT Pharmacom Syst Pharmacol 6:49-57

24. Bergstrand M, Hooker AC, Wallin JE, Karlsson MO (2011) Prediction-corrected visual predictive checks for diagnosing nonlinear mixed-effects models. AAPS J 13:143-151
25. Bajaj G, Gupta M, Feng Y, Statkevich P, Roy A (2017) Exposureresponse analysis of nivolumab in patients with previously treated or untreated advanced melanoma. J Clin Pharmacol 57:1527-1533

26. Liu C, Yu J, Li H, Liu J, Xu Y, Song P, Liu Q, Zhao H, Xu J, Maher VE, Booth BP, Kim G, Rahman A, Wang Y (2017) Association of time-varying clearance of nivolumab with disease dynamics and its implications on exposure response analysis. Clin Pharmacol Ther 101:657-666

27. Zhao X, Suryawanshi S, Hruska M, Feng Y, Wang X, Shen J, Vezina HE, McHenry MB, Waxman IM, Achanta A, Bello A, Roy A, Agrawal S (2017) Assessment of nivolumab benefit-risk profile of a $240-\mathrm{mg}$ flat dose relative to a $3-\mathrm{mg} / \mathrm{kg}$ dosing regimen in patients with advanced tumors. Ann Oncol 28:2002-2008 